\title{
Multifocal Aggressive Squamous Cell Carcinomas Induced by Prolonged Voriconazole Therapy: A Case Report
}

\author{
C. Morice, ${ }^{1}$ A. Acher, ${ }^{1}$ N. Soufir, ${ }^{2}$ M. Michel, ${ }^{1}$ F. Comoz, ${ }^{3}$ D. Leroy, ${ }^{1}$ and L. Verneuil ${ }^{1,4}$ \\ ${ }^{1}$ Department of Dermatology, CHU Caen, Avenue Georges Clémenceau, 14033 Caen, France \\ ${ }^{2}$ Laboratory of Biochemistry and Genetic, Hôpital Bichat, 75018 Paris, France \\ ${ }^{3}$ Department of Pathology, CHU Caen, 14033 Caen, France \\ ${ }^{4}$ Inserm, U728, Paris 75018, France \\ Correspondence should be addressed to L. Verneuil, verneuil-1@chu-caen.fr
}

Received 1 August 2010; Accepted 15 November 2010

Academic Editor: Yolanda T. Becker

Copyright $\odot 2010$ C. Morice et al. This is an open access article distributed under the Creative Commons Attribution License, which permits unrestricted use, distribution, and reproduction in any medium, provided the original work is properly cited.

Voriconazole is a treatment for severe fungal infections. Prolonged voriconazole therapy may induce skin reactions, with $1 \%$ of severe photosensitivity accidents. Recently the imputability of voriconazole in skin carcinogenesis has been suggested. This report concerns a 55-year-old man suffering from pulmonary aspergillosis who presented a phototoxic reaction a few months after introduction of voriconazole, followed by multiple squamous cell carcinomas of sun-exposed skin areas. After voriconazole discontinuation, no new carcinoma was observed. The detection of EBV and HPV in skin lesions was negative. Exploration of gene mutations involved in skin carcinogenesis showed two variants of the MICR gene. The occurrence of multiple, recurrent, aggressive squamous cell carcinomas is rare with voriconazole, but its imputability is strongly suggested. A plausible hypothesis is that several factors including voriconazole uptake, immunosuppression, and genetic background could explain the phenotype of fast-developing skin carcinomas. Voriconazole therapy should be accompanied by stringent photoprotection and skin monitoring.

\section{Introduction}

Voriconazole, a new broad-spectrum triazole antifungal agent, was approved by the US Food and Drug Administration in May 2002. Its use has increased steadily because of its high effectiveness against a wide variety of yeasts and molds and its excellent oral bioavailability [1]. The most common side effects reported are visual disturbances, elevation in hepatic enzyme levels, and rashes $[2,3]$. Photosensitivity reactions of varying severity are frequent $(1 \%)$ and well documented [4-6]. In contrast, only 16 cases of squamous cell carcinomas have been reported, characterized by clinical severity [7-11].

We report a new case of phototoxicity associated with multiple squamous cell carcinomas in a patient treated for 2 years using voriconazole. We discuss the possible mechanisms induced by voriconazole, a photosensitizing drug, in skin carcinogenesis.

\section{Case Presentation}

A man of 55 had been treated with corticosteroids ( $10 \mathrm{mg} /$ day) for sarcoidosis since 1974. In February 2004, treatment comprising $200 \mathrm{mg}$ voriconazole twice daily was instated for pulmonary aspergillosis in this setting of immunosuppression. His usual treatment consisted of salbutamol, budesonide, and ramipril. He had a Fitzpatrick skin phototype II and was living in a temperate area. He was an office worker and had never had intensive, prolonged sunexposure. No previous personal or familial skin cancer was noted.

In May 2004, four months after the voriconazole instatement, a photo-exposure erythema appeared on the face, scalp, and backs of hands, with several relapses. In August 2006, while the patient was still receiving voriconazole, multiple erosive and keratosic lesions of photo-exposed areas appeared, associated with erythema of the back of hands 
(Figure 1(a)). To characterize the photoreaction, phototests were performed. UVA minimum erythema dose (MED) was normal, and UVB-MED was slightly lowered $\left(46,20 \mathrm{~mJ} / \mathrm{cm}^{2}\right.$; $\mathrm{N}>50 \mathrm{~mJ} / \mathrm{cm}^{2}$ ). Photopatch-tests performed in the hypothesis of a photoallergy were negative. These results argued for a phototoxic rather than a photoallergic photosensitive reaction.

The lesions worsened, becoming infiltrated and crusted. The first biopsy on the face in May 2007 showed a microinvasive squamous cell carcinoma. Subsequently, 17 squamous cell carcinomas and multiple actinic keratoses (Figures 1(b) and 1(c)) on the face and scalp were diagnosed up to December 2008 at which time voriconazole was discontinued. Between January and December 2009, actinic keratoses were observed, but no carcinoma. Both curative and preventive treatments associating imiquimod, cryotherapy, photodynamic therapy, and surgery by excision-graft of micro-invasive carcinomas were implemented.

Using in situ hybridation we tested for Epstein-BarrEncoded-small-RNAs- (EBERs-) positive cells with EBVencoded RNA 1 oligonucleotide probe (Benchmark XT automat, Ventana), and for human papillomavirus using reagents and INFORM HPV III Family 16 DNA Probe (B) (P/N 800-4295) (Ventana Medical Systems Inc.), in the cutaneous lesions. No EBV or HPV infection was detected.

Given the phenotype of the multiple skin carcinomas, a search for DNA mutations in genes involved in predisposition to cutaneous carcinoma (MC1R, POLH) was performed. After signature of informed consent and obtaining blood samples, DNA extraction was performed, followed by amplification using PCR primers specific to the coding exons, flanking intrinsic sequences, and automated sequencing (Applied Biosystems $3130^{\mathrm{R}}$ ). No mutation of the POLH (XPV) gene, involved in xeroderma pigmentosum variant, was detected, but two variants of the $M C 1 R$ gene, Arg151Cys and Ser131Asn, were characterized. The first is a well-known loss of function in the $M C 1 R$ variant that is associated with both the RHC (Red Hair Color) phenotype and an increased risk of skin cancer. The second variant, $\mathrm{S} 131 \mathrm{~N}$, has not been previously described, to our knowledge; it occurs at a highly conserved residue and is predicted to be deleterious genomic mutation (SIFT, Polyphen).

\section{Discussion}

Voriconazole, a broad-spectrum azole antifungal agent, inhibits the cytochrome P450-dependent $14 \alpha$-lanosterol demethylation, an essential step in fungal cell membrane ergosterol synthesis $[1,3]$. It is indicated in different fungal infections, particularly invasive aspergillosis [3]. This molecule is easily used by oral route with excellent bioavailability. It is therefore widely prescribed. However, significant side effects have been reported, with 30\% visual disturbances, $10 \%$ liver abnormalities, and $8.6 \%$ skin reactions [1-3].

Among adverse cutaneous drug reactions, cases of generalized erythema, eczema, urticaria, bullous lichen, erythema multiform $(0.05 \%)$, or Lyell's syndrome $(0.1 \%)$ have been reported [1-3]. Photosensitivity, idiosyncratic rather than dose-dependent, is described in $1 \%$ of subjects and appears in prolonged treatment (at least 12 weeks). In most cases, skin reactions disappear on an average of 4 weeks after voriconazole discontinuation $[4,5,12,13]$.

In particular, as in this case report, 15 patients presenting multiple squamous carcinomas have been recently reported in three cases and two series [7-11]. These 15 patients and the present case developed a phototoxic rash on sunexposed areas on an average of 6.56 months after starting treatment ( 15 days to 15 months). The actinic keratoses and squamous cell carcinomas that appeared after an average of 28 months of treatment (12 to 36 months) were multiple. Skin carcinoma onset occurred at a mean age of 38 years ( 9 to 69 years), much earlier compared to the average age for occurrence of these lesions in the general population (76 years) [14]. Among these 16 patients, voriconazole discontinuation led to photosensitivity regression from day 14 for 7 patients and data were unavailable for 9 patients. No new carcinoma had occurred for 4 patients at 10 months after voriconazole discontinuation, and data were unavailable for 12 patients.

Immunosuppression, present in all patients treated with voriconazole, could be expected to induce these squamous cell carcinomas, as in transplant patients [15]. In the setting of immuno-suppression, EBV and HPV have also been implicated in development of nonmelanoma skin cancer [16-18]. In our case, EBV or HPV infection in skin lesions was not detected.

The absence of new squamous cell carcinomas concomitant with the interruption of voriconazole suggests its causal role, especially given its phototoxicity. Two mechanisms are suggested in voriconazole phototoxicity: (i) direct phototoxicity by voriconazole or its metabolites, and (ii) a retinoid-like mechanism $[4,13]$. Like fluoroquinolones, which are also phototoxic molecules, voriconazole may amplify local, systemic immuno-suppression [19] and the genotoxicity [20] induced by UV. An amplification of immuno-suppression by local enhancement of the inhibitory effect on immuno-competent cells has been shown for fluoroquinolones [21]. Concerning genotoxicity, it has been reported, in an experimental model, that fluoroquinolones, especially lomefloxacin and fleroxacin, associated with UVA radiation, enhanced tumor prevalence and drastically shortened the median latent period of tumor onset compared with UVA alone [22-24]. In UV-irradiated xeroderma pigmentosum group A gene-deficient mice, defective in nucleotide excision repair, the treatment with lomefloxacin induced DNA damage [25]. In our case, no mutation of POLH, a gene predisposing to xeroderma pigmentosum variant, was identified. However, two $M C 1 R$ functional variants, Arg151Cys and Ser131Asn, were identified. These variants have been shown to be involved in basal and squamous cell carcinoma [26].

A plausible hypothesis is that several factors including voriconazole uptake, immuno-suppression, and the genetic background ( $M C 1 R$ variants) could explain the phenotype of proliferating multiple skin carcinomas. 


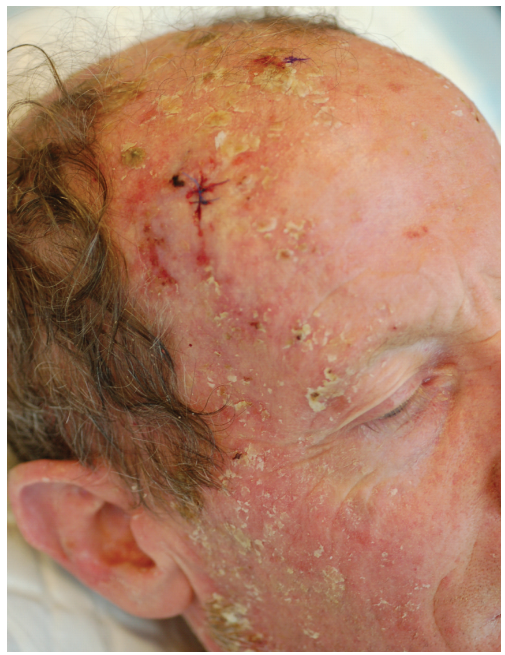

(a)
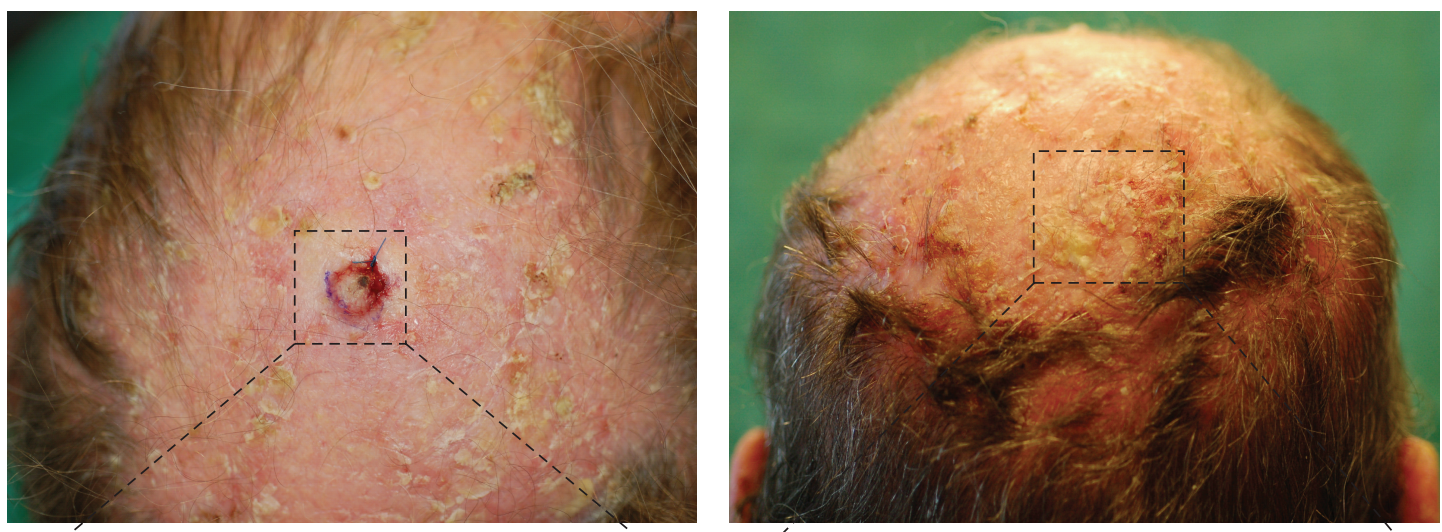

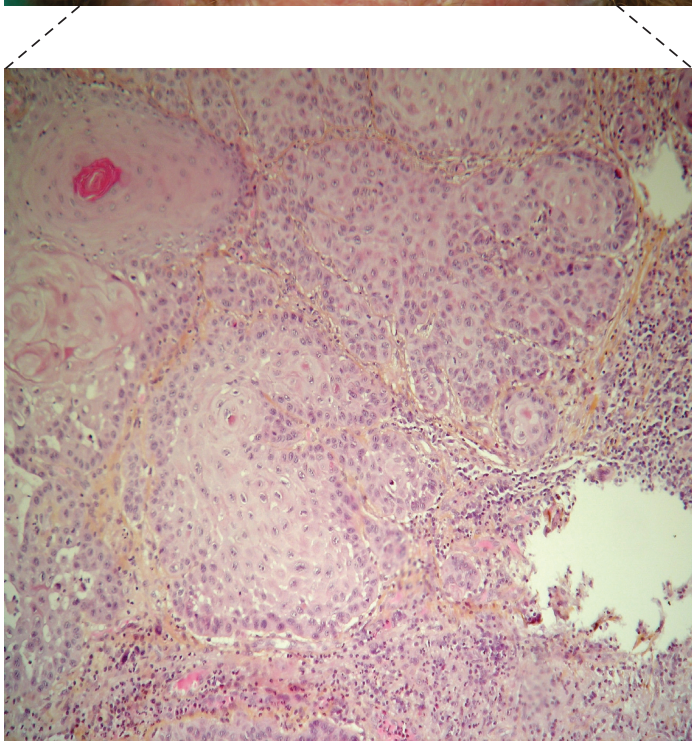

(b)

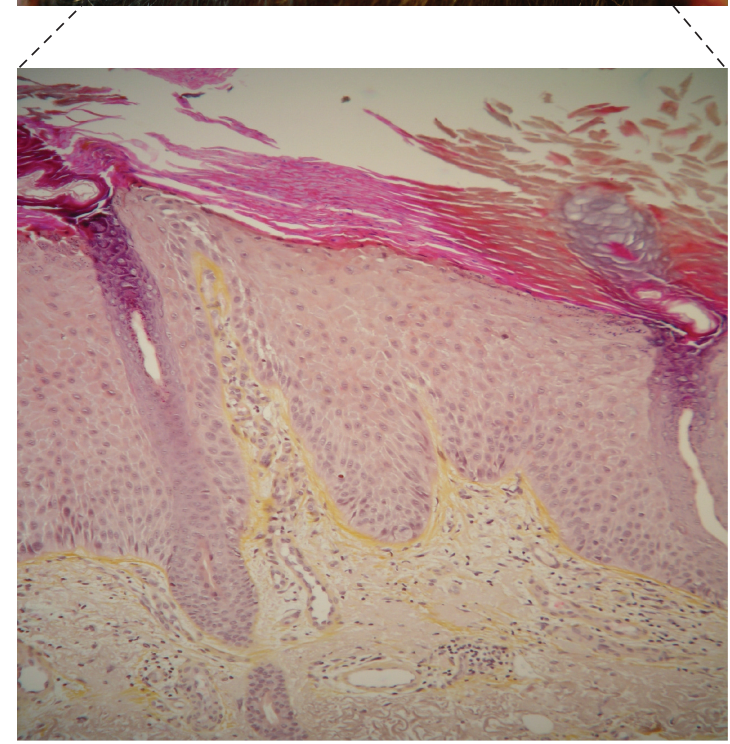

(c)

Figure 1: (a) Actinic keratosis and multiple squamous cell carcinomas of photo-exposed areas induced by voriconazole. (b) Microinvasive squamous cell carcinoma. Epithelial proliferation composed of atypical keratinocytes infiltrating the dermis, magnification $\times 200$. (c) Actinic keratosis hyperkeratosis, slightly atypical epidermal hyperplasia; magnification $\times 200$. 


\section{Conclusion}

Voriconazole, a new antifungal agent, is attractive by its efficiency and ease of use. However, our case and others in the literature suggest that prolonged treatments, in a context of immunosuppression complicated by phototoxicity reactions, can predispose to risk of multiple recurrent, aggressive squamous cell carcinomas. In patients at risk, an alternative therapy should be discussed whenever possible. Dermatological supervision, stringent measures of external photoprotection, and sun avoidance are recommended.

\section{Conflict of Interests}

The authors declare that they have no conflict of interests.

\section{Authors' Contribution}

C. Morice, L. Verneuil, and D. Leroy contributed in writing the paper and reviewing of the literature as well as undertaking a comprehensive literature search; A. Acher, M. Michel, F. Comoz, and N. Soufir helped with the acquisition of data and manuscript revision.

\section{References}

[1] L. Jeu, F. J. Piacenti, A. G. Lyakhovetskiy, and H. B. Fung, "Voriconazole," Clinical Therapeutics, vol. 25, no. 5, pp. 13211381, 2003.

[2] A. E. Boyd, S. Modi, S. J. Howard, C. B. Moore, B. G. Keevil, and D. W. Denning, "Adverse reactions to voriconazole," Clinical Infectious Diseases, vol. 39, no. 8, pp. 1241-1244, 2004.

[3] L. B. Johnson and C. A. Kauffman, "Voriconazole: a new triazole antifungal agent," Clinical Infectious Diseases, vol. 36, no. 5, pp. 630-637, 2003.

[4] A. N. Malani and D. M. Aronoff, "Voriconazole-induced photosensitivity," Clinical Medicine and Research, vol. 6, no. 2, pp. 83-85, 2008.

[5] N. Auffret, F. Janssen, P. Chevalier, R. Guillemain, C. Amrein, and C. Le Beller, "Voriconazole photosensitivity: 7 cases," Annales de Dermatologie et de Venereologie, vol. 133, no. 4, pp. 330-332, 2006.

[6] A. J. Racette, H. H. Roenigk, R. Hansen, D. Mendelson, and A. Park, "Photoaging and phototoxicity from long-term voriconazole treatment in a 15-year-old girl," Journal of the American Academy of Dermatology., vol. 52, no. 5, pp. S81-S85, 2005.

[7] K. L. McCarthy, E. G. Playford, D. F. Looke, and M. Whitby, "Severe photosensitivity causing multifocal squamous cell carcinomas secondary to prolonged voriconazole therapy," Clinical Infectious Diseases, vol. 44, no. 5, pp. e55-e56, 2007.

[8] A. Vanacker, G. Fabré, J. Van Dorpe, W. E. Peetermans, and B. Maes, "Aggressive cutaneous squamous cell carcinoma associated with prolonged voriconazole therapy in a renal transplant patient," American Journal of Transplantation, vol. 8, no. 4, pp. 877-880, 2008.

[9] A. S. Brunel, T. Fraisse, C. Lechiche, V. Pinzani, J. M. Mauboussin, and A. Sotto, "Multifocal squamous cell carcinomas in an HIV-infected patient with a long-term voriconazole therapy," AIDS, vol. 22, no. 7, pp. 905-906, 2008.
[10] O. Epaulard, C. Saint-Raymond, C. Villier et al., "Multiple aggressive squamous cell carcinomas associated with prolonged voriconazole therapy in four immunocompromised patients," Clinical Microbiology and Infection, vol. 16, no. 9, pp. 1362-1364, 2010.

[11] E. W. Cowen, J. C. Nguyen, D. D. Miller et al., "Chronic phototoxicity and aggressive squamous cell carcinoma of the skin in children and adults during treatment with voriconazole," Journal of the American Academy of Dermatology, vol. 62, no. 1, pp. 31-37, 2010.

[12] C. K. Dolan, M. A. Hall, D. L. Blazes, and C. W. Norwood, "Pseudoporphyria as a result of voriconazole use: a case report," International Journal of Dermatology, vol. 43, no. 10, pp. 768-771, 2004.

[13] M. Rubenstein, M. L. Levy, and D. Metry, "Voriconazoleinduced retinoid-like photosensitivity in children," Pediatric Dermatology, vol. 21, no. 6, pp. 675-678, 2004.

[14] L. Martin and J. J. Bonerandi, "Carcinome épidermoïde cutané (carcinome spinocellulaire) recommandations de pratique clinique pour la prise en charge diagnostique et thérapeutique," Annales de Dermatologie et de Venereologie, vol. 136, no. 5, pp. S163-S164, 2009.

[15] S. Euvrard, J. Kanitakis, and A. Claudy, "Cutaneous tumors in organ transplant recipients," Presse Medicale, vol. 31, no. 40, pp. 1895-1903, 2002.

[16] A. Zaravinos, P. Kanellou, and D. A. Spandidos, "Viral DNA detection and RAS mutations in actinic keratosis and nonmelanoma skin cancers," British Journal of Dermatology, vol. 162, no. 2, pp. 325-331, 2010.

[17] A. Ternesten-Bratel, C. Kjellström, and A. Ricksten, "Specific expression of Epstein-Barr virus in cutaneous squamous cell carcinomas from heart transplant recipients," Transplantation, vol. 66, no. 11, pp. 1524-1529, 1998.

[18] L. Struijk, E. van der Meijden, S. Kazem et al., "Specific betapapillomaviruses associated with squamous cell carcinoma of the skin inhibit UVB-induced apoptosis of primary human keratinocytes," Journal of General Virology, vol. 89, no. 9, pp. 2303-2314, 2008.

[19] M. Norval, "The mechanisms and consequences of ultravioletinduced immunosuppression," Progress in Biophysics and Molecular Biology, vol. 92, no. 1, pp. 108-118, 2006.

[20] D. S. Rigel, "Cutaneous ultraviolet exposure and its relationship to the development of skin cancer," Journal of the American Academy of Dermatology, vol. 58, no. 5, pp. S129S132, 2008.

[21] K. Murata, K. Sugita, M. Kobayashi, K. Kabashima, and Y. Tokura, "Nadifloxacin downmodulates antigen-presenting functions of epidermal Langerhans cells and keratinocytes," Journal of Dermatological Science, vol. 42, no. 2, pp. 91-99, 2006.

[22] V. Lhiaubet-Vallet, F. Bosca, and M. A. Miranda, "Photosensitized DNA damage: the case of fluoroquinolones," Photochemistry and Photobiology, vol. 85, no. 4, pp. 861-868, 2009.

[23] G. Klecak, F. Urbach, and H. Urwyler, "Fluoroquinolone antibacterials enhance UVA-induced skin tumors," Journal of Photochemistry and Photobiology B, vol. 37, no. 3, pp. 174-181, 1997.

[24] B. E. Johnson, N. K. Gibbs, and J. Ferguson, "Quinolone antibiotic with potential to photosensitize skin tumorigenesis," Journal of Photochemistry and Photobiology B, vol. 37, no. 3, pp. 171-173, 1997.

[25] T. Itoh, H. Miyauchi-Hashimoto, A. Sugihara, K. Tanaka, and T. Horio, "The photocarcinogenesis of antibiotic lomefloxacin 
and UVA radiation is enhanced in xeroderma pigmentosum group A gene-deficient mice," Journal of Investigative Dermatology, vol. 125, no. 3, pp. 554-559, 2005.

[26] N. F. Box, D. L. Duffy, R. E. Irving et al., "Melanocortin-1 receptor genotype is a risk factor for basal and squamous cell carcinoma," Journal of Investigative Dermatology, vol. 116, no. 2, pp. 224-229, 2001. 


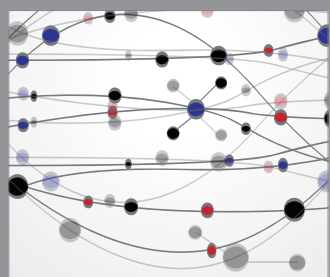

The Scientific World Journal
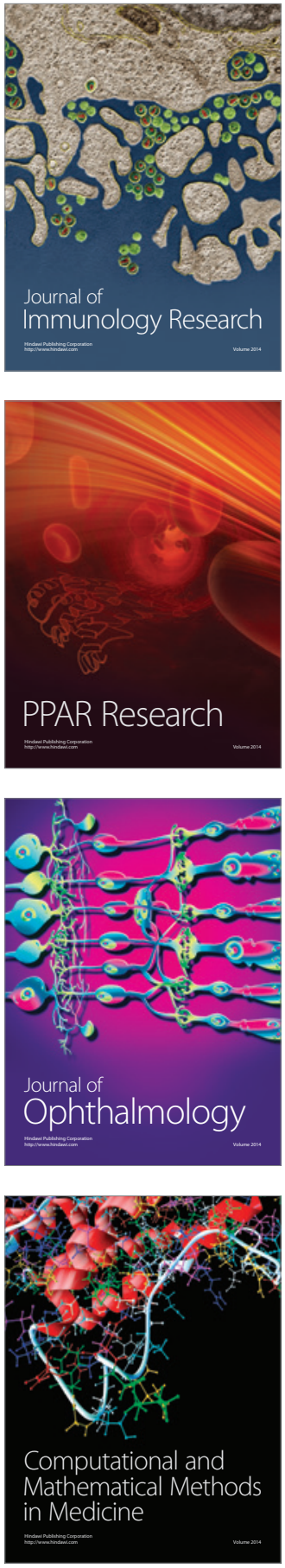

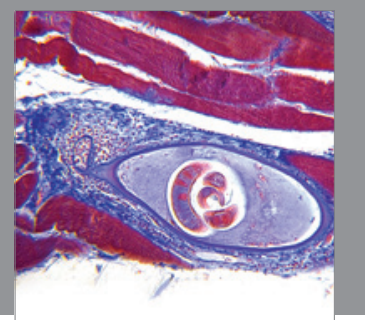

Gastroenterology

Research and Practice
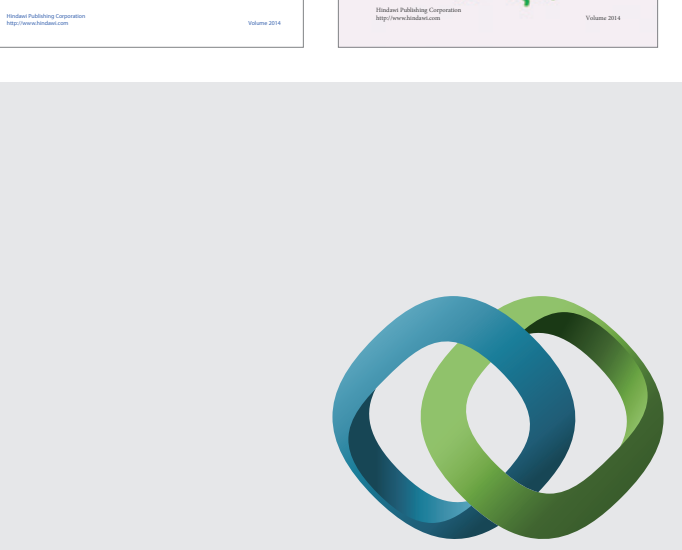

\section{Hindawi}

Submit your manuscripts at

http://www.hindawi.com
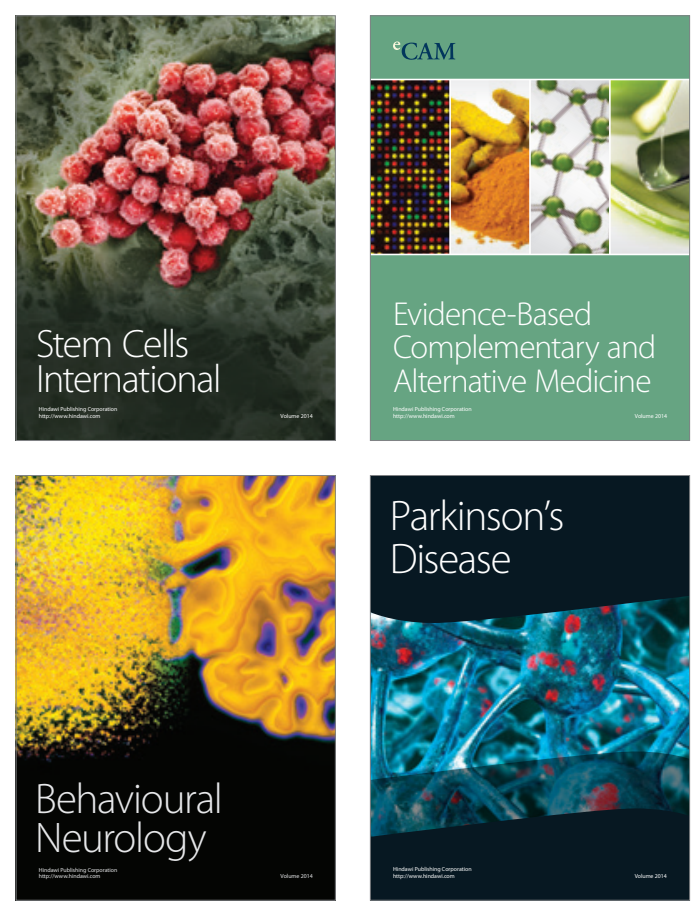

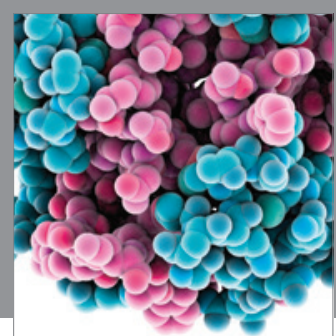

Journal of
Diabetes Research

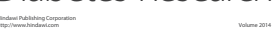

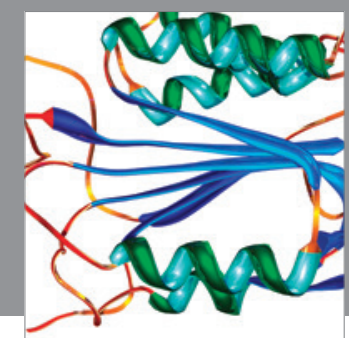

Disease Markers
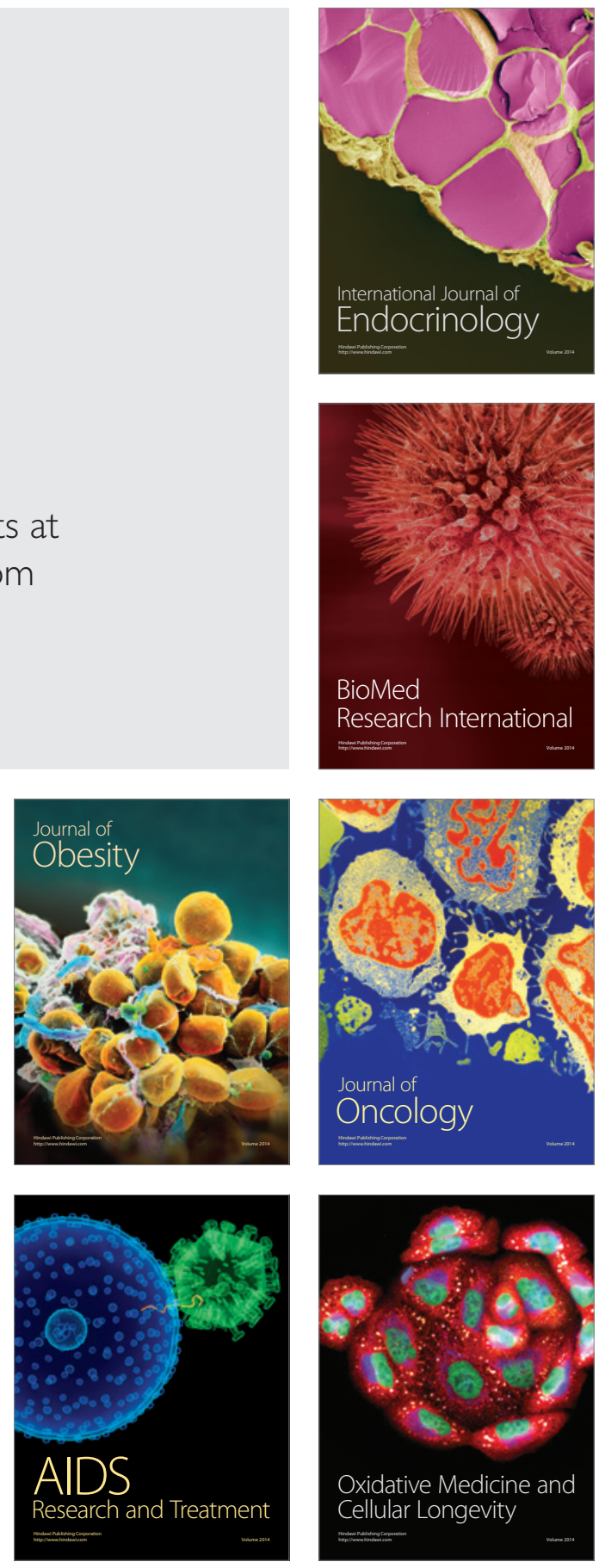